\title{
Innovación abierta: de consumidores a productores de valor
}

\author{
CARMEN BUENO CASTELLANOS
}

En este ensayo se presentan las diferencias y similitudes que se encontraron en dos convocatorias abiertas, llamadas crowdsourcing, lanzadas por firmas globales que producen bienes de consumo con la finalidad de cooptar ideas creativas de la sociedad. Este ejercicio pretende analizar la manera en la que el valor es producido por la participación activa de un nuevo actor social, denominado prosumidor, y después expropiado por las firmas. Se pretende contribuir al entendimiento de cómo formas inéditas de producción de conocimiento, mediadas por las tecnologías de la información, han impactado en el proceso de acumulación de capital, así como en el valor estratégico de los datos y los proyectos de innovación abierta que fluyen en el espacio virtual.

PALABRAS CLAVE: capitalismo cognitivo, crowdsourcing, prosumidor, marca, economía afectiva

Open Innovation: From Cosumers to Producers of Value

This essay presents the differences and similarities found in two crowdsourcings launched by global firms that produce consumer goods in order to co-opt creative ideas from society. This exercise intends to analyze the way value is produced by the active participation of a new social actor, which is given the name of prosumer, and thereafter appropriated by these firms. Consecuently contributing to the understanding of how unprecedented ways of knowledge production mediated by information technologies have impacted the process of capital accumulation, as well as the strategic value of data and open innovation projects that flow through the virtual space.

KEYWORDS: cognitive capitalism, crowdsourcing, prosumer, brand, emotional economy 


\section{Presentación del tema'}

as firmas productoras de bienes de consumo que dominan la economía mundial enfrentan el gran desafío de mantener un proceso constante de innovación. La intensa competencia las incita a crear múltiples mecanismos para conservar la lealtad de sus clientes y abrir nuevos mercados. La generación de productos con la participación activa de los compradores es estratégica. Desouza et al. (2008) denominan este proceso "innovación impulsada por los consumidores", porque estos se convierten en productores de nuevas ideas y a ellos se suman los seguidores o fans de las marcas, quienes expresan sus preferencias por medio de redes sociales.

Estos cambios son una reacción a los fuertes costos de operación de los departamentos de investigación y desarrollo que bajo el modelo fordista, e incluso en las primeras etapas de la producción flexible, habían operado con gran hermetismo. Gassman, Sandmeier y Wecht (2006) afirman que la creación de ideas operaba con prácticas de alta cerrazón. ${ }^{3}$ Este primer impulso creativo significaba hasta $85 \%$ del costo del desarrollo de un nuevo producto, además de que los ciclos del proceso innovador resultaban largos. Habría que añadir que el riesgo de una aceptación poco exitosa en el mercado era muy alto, pues las opiniones de los consumidores, obtenidas con técnicas como grupos focales o encuestas, no se aplicaban sino hasta que el producto estaba por lanzarse al mercado.

$1 \quad$ En este ensayo se optó por mantener los anglicismos que se utilizan en las plataformas de innovación abierta. Estos términos en inglés se han convertido en la lingua franca que permite homogeneizar la comunicación en dichos espacios. Su traducción y explicación se presentan en notas a pie de página.

2 El concepto, en su versión original, es costumer driven innovation. Los autores presentan múltiples mecanismos, entre ellos, la innovación centrada en el consumidor, la innovación del consumidor por los consumidores y la innovación dinámica dirigida a mercados segmentados.

3 El concepto en inglés es in-lock. 
Ante estas desventajas, las grandes corporaciones han recompuesto sus estrategias para producir innovaciones. Una de las más significativas es lo que los autores que hemos citado llaman interfaz de la innovación extrema centrada en el cliente. ${ }^{4}$ Esto implica desarrollar puntos de intersección con los usuarios vigentes y potenciales y convocarlos a una participación activa en el lanzamiento de ideas creativas, abrir "rendijas" que permitan un contacto directo con los consumidores, dondequiera que se encuentren, y sacar provecho de la información que ofrecen de manera voluntaria.

La innovación abierta ${ }^{5}$ es un proceso que ha crecido de manera exponencial a partir del desarrollo de canales de conectividad que dan cabida a actividades relacionales en las que se comparte información y se produce conocimiento. Es un nuevo ensamble que toma formas variadas, todas dinámicas, difusas, difíciles de percibir a simple vista, pues operan dentro del espacio virtual. El manejo adecuado de los recursos que fluyen a través de la web supone grandes desafíos para las firmas, sobre todo en su capacidad de coordinación y en su control sobre el proceso.

La innovación abierta opera mediante mecanismos numerosos. En este ensayo se seleccionó el crowdsourcing. ${ }^{6}$ En la actualidad, toda firma de prestigio lanza estas convocatorias masivas para atraer las ideas de la "clase creativa" (Florida, 2010). En diversos circuitos que integran el espacio de flujos (Castells, 2006), se invita a proponer ideas creativas para la solución de problemas o el desarrollo de productos nuevos. Los participantes ofrecen un reservorio de propuestas que surgen de sus experiencias de consumo y pericia profesional, y se convierten en una extensión coyuntural de la firma al mismo tiempo que abonan a la construcción del mundo corporativo al que aluden Welker, Partridge y Har$\operatorname{din}(2011)$.

La firma se encarga de seleccionar las propuestas que pueden ser explotadas según sus intereses, ya sea al integrar comisiones ad hoc entre empleados con posiciones estratégicas de manufactura, mercado o finanzas; invitar a empleados o directivos retirados, que conocen la firma desde dentro pero ya no tienen una posición de dirección, o a personas externas que tienen algún vínculo con la firma. Otro recurso son los algoritmos que codifican y agrupan el cúmulo de datos que fluyen en la web.

Para explicar cómo opera la innovación abierta, se presentan dos programas de crowdsourcing de firmas que producen bienes de consumo con importantes componentes tecnológicos en sus productos, insumos y procesos de manufactura y distribución, como automóviles y productos para el hogar y la higiene personal, que además tienen una gran presencia mundial. A partir de estos casos, se pretende averiguar cómo se produce y cómo se expropia el valor de la participación activa de un nuevo actor social, al que se ha dado el nombre de prosumidor. ${ }^{7}$

Para contestar esta pregunta, el siguiente apartado introduce una reflexión sobre la manera en la que las nuevas relaciones de producción de

4 El concepto, en la versión original, es extreme customer innovation in the front-end.

$5 \quad$ Los procesos de innovación abierta no son privativos de las grandes firmas, muchos gobiernos, organizaciones no gubernamentales, fundaciones y organismos internacionales utilizan estos mecanismos para convocar a la ciudadanía a presentar soluciones a problemas sociales y ambientales. Este ensayo sólo presenta dos casos desarrollados por grandes firmas.

$6 \quad$ La traducción al español es "colaboración abierta distribuida", sin embargo, los millennials usan el término en inglés. Los millennials son una generación que nació en las décadas de 1980 y 1990; se les considera "nativos digitales" porque a partir de ese momento surgen y se desarrollan tecnologías que permiten la conectividad y la comunicación virtuales.

7 El concepto fue creado por un futurista llamado Toffler, en su libro The Third Wave, escrito en 1980, editado por la casa Bantam. Es un acrónimo que fusiona la palabra producer y consumer. También se asocia profesional a consumer (Arvidsson y Colleoni, 2012). 


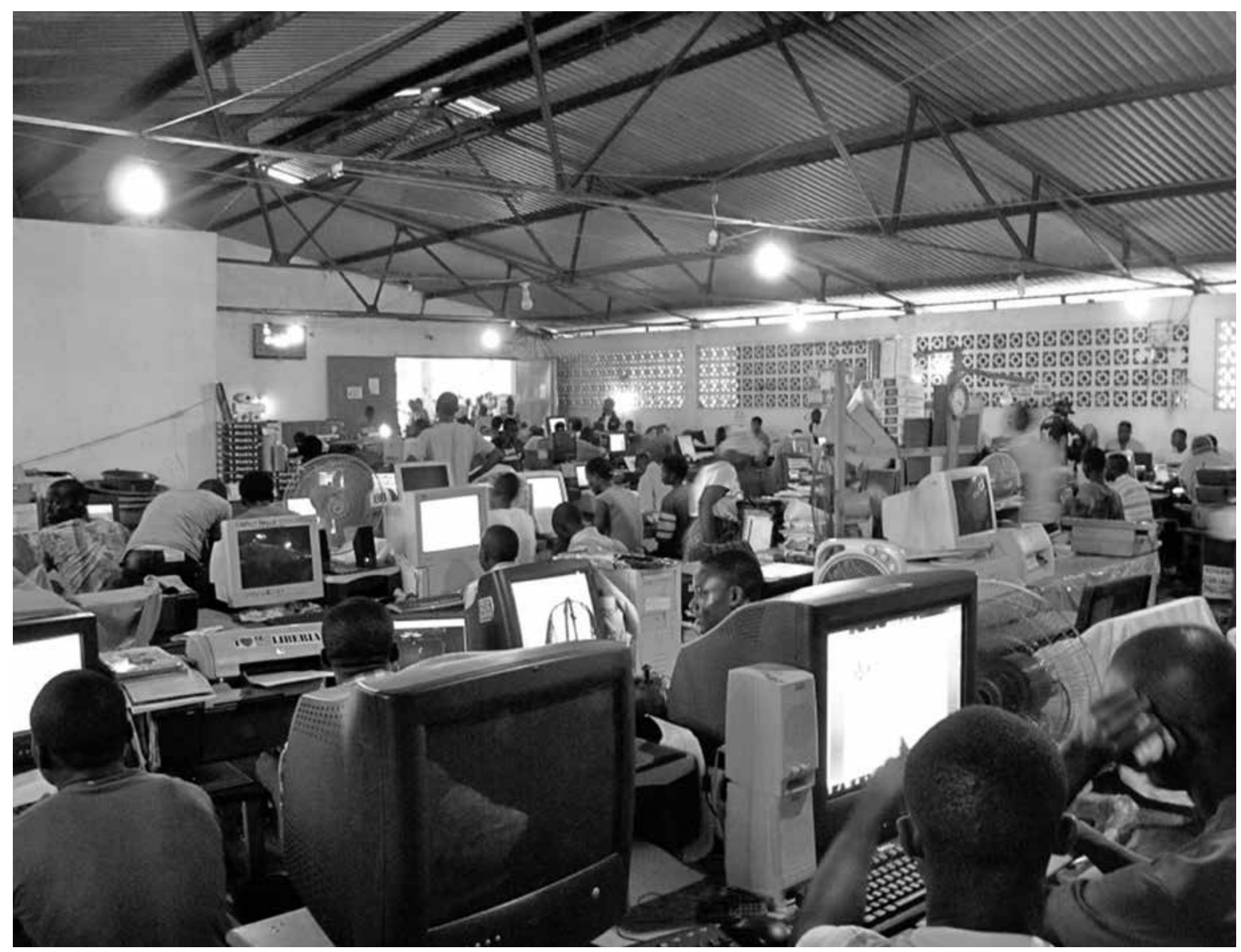

FRANCO PAPESCHI - Una mezcla entre co-working y mercado en el World Trade Center de Monrovia, Liberia, mayo de 2012.

conocimiento, mediadas por las tecnologías de la información, han impactado en los procesos de acumulación de capital, así como en el valor estratégico de los datos y las propuestas creativas generadas por los prosumidores. Después se presenta la reconstrucción de dos casos de crowdsourcing que ilustran la importancia que estas convocatorias tienen para el posicionamiento de la marca. El apartado final hace un ejercicio comparado de las diferencias y similitudes entre los casos expuestos en cuanto a la manera en que se produce y se expropia valor en estas estrategias de innovación abierta.

Para seleccionar los dos casos de crowdsourcing se tomó en cuenta que fueran pioneros en el lanzamiento de estas convocatorias abiertas y que reflejaran propósitos y dinámicas diferentes. Otro elemento importante fue que se pudiera rastrear un ciclo completo del proceso para apreciar los mecanismos de cocreación de innovaciones en un espacio abierto y que tuviera suficiente información de acceso libre. ${ }^{8}$ Todos los datos que sustentan estos casos se recuperaron en el espacio en el que operan y se comunican los prosumidores de marcas reconocidas

8

La convocatoria Fiat Mio surge en 2009, y el programa Connect \& Develop, en 2000, pero logra una cierta madurez en 2008. 
en el mundo y que participan en procesos de innovación abierta. ${ }^{9}$ Se revisaron blogs ${ }^{10}$ redes sociales, videos en YouTube ${ }^{11}$ e información que publican las firmas en sus páginas web. Desde esta perspectiva, la investigación a partir de la web franquea el acceso a dinámicas, interacciones e información de actividades que operan principalmente en el mundo virtual. A diferencia de la etnografía presencial, Hine (2000) argumenta que el ciberespacio ha construido un formato de interacción compuesto de textos que son portátiles, susceptibles de ser transportados lejos de las circunstancias que los han originado.

Cabe aclarar que la información recuperada en canales de acceso libre sobre estos temas es por lo regular aquella que la firma ha querido hacer pública, que aporta a su imagen, y por lo tanto, a su estrategia publicitaria. La lectura e interpretación de estos textos e imágenes permitió sostener la pregunta central referida a las logísticas de producción y expropiación de valor de las nuevas estrategias generadoras de innovación.

\section{Crowdsourcing, estrategia inédita en la generación y apropiación de valor}

Las tácticas actuales en la generación y apropiación de valor han dado cabida a la adjetivación del proceso de acumulación. Para comprender el papel que juegan en estos procesos los crowdsourcings impulsados por firmas que producen bienes tangibles para el consumo personal y que operan en un nivel mundial, se presentan los aportes centrales del capitalismo netárquico, propuesto por Rifkin (2000),,$^{12}$ y el capitalismo cognitivo, que se expone en las reflexiones de Arvidsson y Colleoni (2012), Fumagalli (2010) y Moulier-Boutang (2011). El primer tipo de capitalismo da testimonio de la estructura relacional virtualizada necesaria para que fluyan y se compartan ideas y conocimiento, mientras el segundo se centra en el valor otorgado a los bienes inmateriales que anteceden y se incorporan a los productos - jabones de todo tipo y automóviles - que estas firmas colocan en el mercado. Son propuestas complementarias que permiten analizar las relaciones actuales de la producción y apropiación de conocimiento, incrustadas en un nuevo paradigma de relaciones de poder de la economía mundial, lo que ha modificado la correlación de fuerzas y ha agregado connotaciones antes inexistentes a los procesos generadores de riqueza. Lo específico del crowdsourcing como instrumento de innovación abierta es que hay una dislocación entre

9

Se presentan los primeros avances del proyecto titulado "Procesos de innovación en masa: estrategia del mundo conexionista”, financiado por la Dirección de Investigación de la Universidad Iberoamericana por el periodo 2017-2019. Un blog es un sitio web que integra grupos de interés que interactúan y cocrean ideas. Todas las entradas se almacenan en orden cronológico. Además, es un espacio vinculante con otros colectivos similares, que producen de manera exponencial comunidades virtuales que se identifican con un tema o ideología. A quienes crean y hacen uso de blogs se les denomina blogueros.

11 YouTube es un sitio web que permite que los usuarios compartan videos con contenidos diversos.

12 Latour (2005) y Callon (1990), a partir de la década de 1980, fueron precursores del análisis de la estructura en red de los procesos de producción de conocimiento o teoría del actor-red. Estas aportaciones, a pesar de ser importantes referentes en los estudios de la ciencia, la tecnología y la sociedad, no se retoman en este ensayo por tres razones básicas: 1) aquí los artefactos y las técnicas se conciben sólo como facilitadores de relaciones sociales y su función no es equiparable al papel que juegan los actores sociales. Por lo tanto, la tecnología no se entiende como un actante -término utilizado por Latour y Callon- que transmute y mantenga el mismo protagonismo que los humanos en un entramado social "sin costuras". 2) La contribución de estos autores se circunscribe al contexto científico y técnico, y excluye a los consumidores como cocreadores de procesos innovadores. El mercado se comprende como un polo independiente del científico y técnico, por lo tanto, no se incorpora al proceso innovador. 3) La teoría del actorred se centra en el análisis de la conformación de procesos sociotécnicos y no aborda los temas de las implicaciones de la innovación en red abierta para la generación y apropiación de valor en la lógica capitalista. 
el espacio de creación/extracción de valor y el de su apropiación.

El capitalismo netárquico analiza las implicaciones que tiene el control sobre los vectores y algoritmos, que se han convertido en medios de producción cuya función es dar seguimiento, capturar, organizar y codificar el cúmulo infinito de datos — big data - que fluye en el espacio virtual y rebasa la capacidad humana para su procesamiento. Estos mecanismos lógico-matemáticos se han convertido en potentes habilitadores de procesos que van cada vez más rápido, con mayor facilidad, de manera ubicua y libre. El control de esta infraestructura otorga a las firmas el derecho de adjudicación de la información que circula de manera desprotegida o con protección alternativa a los derechos de autor. Por ello, este tipo de capitalismo desarrolla una reconfiguración de los nodos estratégicos y otorga una posición hegemónica a las firmas que cooptan datos que reportan valor agregado a la economía mundial.

Las firmas de tecnología se convierten en socios estratégicos de las firmas que producen bienes de consumo. Éstas requieren el apoyo tecnológico para sacar el mayor provecho de la producción de conocimiento que se origina off-shore. ${ }^{13}$ El lanzamiento de crowdsourcings no podría tener la escala en la que opera si no fuera por los servicios de empresas que ofrecen múltiples instrumentos mediáticos y virtualizados para que los datos fluyan de manera libre y se organicen según los objetivos e intereses de las empresas. Estas plataformas, además, seleccionan las propuestas propicias para ser explotadas bajo ciertos criterios y almacenan en espacios llamados backlog ${ }^{14}$ los datos que no se usan en ese momento pero que pueden ser aprovechados en el futuro. De esta manera, todos los datos se transforman en recursos que generan patrones de respuesta de los consumidores a escala global y pueden ser reutilizados para propósitos diversos.

La dupla de firmas que tiene un posicionamiento hegemónico en el mercado mundial reconoce las ventajas competitivas de romper barreras geográficas, sociales y étnicas para dar oportunidad a que el talento se exprese y aporte algo, dondequiera que se encuentre. El flujo de información en red toma formas variadas, conecta personas y codifica datos que reflejan gustos, preferencias y emociones de manera mediática. De esta manera, el espacio en el que tiene lugar la innovación abierta se integra por hardware — dimensión física —, software — dimensión lógicay wetware — dimensión cerebral y vivencial de la muchedumbre creativa que autorregula la producción de conocimiento- . No es fortuito que a internet se le haya denominado "el bien común global de la inteligencia colectiva” (Moulier-Boutang, 2011: 26).

Riftkin (2000), igual que Moulier-Boutang (2011), argumenta que el capitalismo netárquico genera cuantiosas ganancias al estructurar una red de redes que facilita el libre flujo de bienes comunes colaborativos como resultado de formas inéditas de proximidad con los consumidores, y recrea un sinnúmero de direccionalidades y vías de conectividad y encuentro con la sociedad (Rifkin, 2000: 9). No se trata de plataformas tecnológicas y esquemas matemáticos que facilitan de manera neutral la captación y ordenamiento de los datos, sino de fuentes de poder que perfilan el control sobre la toma de decisiones de los procesos innovadores externalizados de las firmas y contribuyen al posicionamiento de las marcas en el mercado financiero.

Acerca de la discusión central en la conceptualización del capitalismo cognitivo, Arvidsson y Colleoni (2012), Fumagalli (2010) y Moulier-Boutang (2011) coinciden en afirmar que los datos que fluyen libremente en la web, producidos por consumidores y cooptados por múltiples redes sociales, son los que

13 Off-shore es la producción de ideas en espacios externos a las firmas.

14 Backlog es una bitácora de respaldo, almacenada y a disposición exclusiva de la firma. 
se han convertido en la mercancía —intangibleque otorga valor a la marca en los centros financieros. En esta perspectiva, ya no es la capacidad productiva de la firma sino la reputación de la marca en las bolsas de valores internacionales lo que predomina en la lógica de acumulación de las empresas que controlan el cúmulo de información que circula en el espacio de los flujos. Esta reputación se sustenta en la capacidad de las firmas de generar y ampliar relaciones afectivas con los consumidores reales y potenciales, como argumentan estos autores, cuyo aporte central sólo se esboza aquí porque permite reflexionar acerca del papel que juega el crowdsourcing en el fortalecimiento de la reputación de la marca.

Para las grandes firmas, la marca es el elemento diferenciador y su principal vínculo con los consumidores. La carga simbólica de la marca se ha convertido en su principal ventaja económica. Por ello, las firmas tienen que generar mecanismos diversos de mercadotecnia con estrategias persuasivas para mantener su reputación. Esta acción se ha convertido en un bien intangible "líquido", denominado audience commodity (Smythe, citado en Arvidsson y Colleoni, 2012: 138). ${ }^{15}$ Su particularidad es expropiar valor de los seguidores de un objeto que aún es conceptual y que fluye por los diversos canales de la web, una estrategia distintiva de la "economía de carnada". ${ }^{16}$ Esta práctica lúdica, imperceptible para el usuario de las redes sociales, fortalece en automático el prestigio y la popularidad de una marca porque su presencia en la web se vuelve exponencial. Los autores citados afirman que los hits ${ }^{17}$ permiten medir el interés que suscita la marca en un ambiente comunicativo virtualizado. El seguimiento cuantitativo de los hits se convierte en el insumo que otorga valor a la marca. Esto, como se mencionó, se produce dentro de la lógica de los mercados financieros y no en los espacios de la producción.

Moulier-Boutang (2011) afirma que ahora más que nunca se está desarrollando una economía de los afectos que fortalece sus mercados al enganchar a los consumidores en experiencias vivenciales de placer, pasión, satisfacción y lealtad a la marca. Como afirma un joven que ha participado en crowdsourcings y convocatorias similares, como los hackathons: ${ }^{18}$ "nosotros los millennials somos emocionales, nos gusta experimentar". Esto demuestra que las cargas afectivas que identifican a los consumidores con la marca son potenciados por las firmas cuando desarrollan eventos cuyo objetivo es brindar experiencias exclusivas y originales. A ello se añade otro elemento que fecunda el prestigio de la marca y también es un bien intangible: la producción de innovaciones que surgen del número infinito de ideas generadas por las "multitudes creativas". Como se verá en los dos casos que se presentan aquí, se trata de materia prima germinada que después de un riguroso proceso de selección se convierte en conocimiento susceptible de ser incorporado en productos de consumo que se venden en mercados mundiales.

La centralidad de la participación activa de las "multitudes creativas" en la lógica de acumulación del capitalismo cognitivo modifica la posición social del consumidor, que pasa de ser un comprador de productos y servicios a convertirse en un "colaborador", es decir, en un productor "externalizado" en el proceso de generación de valor. Al incorporarse a los circuitos de producción online, el consumidor toma un papel activo; por ello, a este

Audience commodity es un producto mercantilizable en el cual la "audiencia", es decir, los consumidores reales y potenciales, participa con su aportación de ideas al proceso innovador.

16 "Economía de carnada" es una de las discusiones que presenta Gustavo Lins Ribeiro en este número de Desacatos.

17 Hit es cuando se pulsa un botón en los teléfonos inteligentes o en las computadoras para demostrar empatía o rechazo hacia una información o imagen.

18 El hackathon es un evento presencial que puede durar hasta 48 horas, en el que equipos de jóvenes desarrollan aplicaciones para resolver un problema social. Algunos llegan a tener 2000 o 3000 participantes. 
actor social se le denomina prosumidor (Reichwald et al., 2003; Arvidsson y Colleoni, 2012; Wittke y Hanekop, 2011). La siguiente reflexión, vertida en una publicación con enfoque de negocios, hace explícito este cambio de perspectiva: "para ser un líder en el mercado, deja que tu consumidor impulse propuestas" (Desouza et al., 2008: 35). En este sentido, el artículo no sólo propone reaccionar a la demanda del mercado, sino alinear la planeación de la organización a las necesidades expresadas por compradores clave, que están abiertos a ofrecer datos "agudos" — mi traducción sería "ideas innovadoras" - para propuestas futuras. Para ello, proponen que las firmas inviertan dinero, esfuerzo y tiempo en abrir espacios para desarrollar competencias de cocreación y asociación, como una manera de reconocer que la integración de recursos externos abona al incremento significativo del valor en el mercado.

Así, el prosumidor se convierte en un actor social estratégico para el lanzamiento de ideas novedosas, cuyo perfil se incluye en el universo de los millennials con visión cosmopolita y flexible. La capacidad creativa de estos actores sociales es el resultado de habilidades de navegación virtual para hacerse de información y generar conocimiento con softwares especializados. A esto se tienen que sumar habilidades sociales y comunicativas en la red que les permiten colaborar, conectarse y participar en comunidades virtuales. Los que sobresalen en estos espacios de cocreación se convierten en trendsetters ${ }^{19}$ (García Canclini y Piedras, 2013), es decir, personas que han aprovechado el desarrollo de sus capacidades para proponer estilos y tendencias novedosas, lo que combinan con su experiencia vivencial como consumidores.

Moulier-Boutang (2011) afirma que hay una transformación de las convenciones que operan en este espacio de cocreación como resultado de la participación del prosumidor. Estas convenciones producidas por la colectividad que participa de manera deliberada o inconsciente en los crowdsourcings son representativas de las formas globales que analizan Ong y Collier (2009), quienes señalan que para comprender y apropiarse de estas convenciones en el plano societal se tiene que producir un conjunto de significados, entendimientos, prácticas y estructuras transmitidas de manera tácita. Son inversiones afectivas que se desprenden de prácticas colaborativas reproducidas en la dinámica de las comunidades de pertenencia. De esta forma, se genera un consentimiento colectivo de los cibernautas que responden a las convocatorias que lanzan las grandes firmas. La demostración de este consenso está en la respuesta masiva que crece de modo exponencial y en la participación de los blogueros en este ambiente virtualizado. Así se ejerce y legitima el control social sobre el espacio de cocreación que opera en las redes virtuales y se convierte en el referente de la forma de actuar de los prosumidores.

Las firmas encargadas de ofrecer plataformas tecnológicas y canales de comunicación y cocreación virtual generan de manera implícita su cultura corporativa. Ésta no sólo moldea su dinámica interna sino la vida social de quienes se identifican con la marca y participan en las comunidades de pertenencia. Las inversiones afectivas de la sociedad consumidora son organizadas por los algoritmos y la minería de datos con la finalidad de intervenir en el valor de los productos que se lanzan al mercado. Las convenciones se convierten en una referencia que permite hacer cálculos de valor financiero de la marca (Arvidsson y Colleoni, 2012).

El papel productivo de los consumidores - reales o potenciales - se incluye en la categoría de "trabajo libre" (Terranova, 2004, citado en Arvidsson y Colleoni, 2012: 137) y forma parte del

19 Trendsetter es un innovador que desarrolla propuestas disruptivas o al menos establece una tendencia a seguir en un tipo de consumo. 
trabajo autogenerado y autorregulado en un proceso colectivo de producción de conocimiento disperso en el espacio de los flujos, y por lo tanto, no supervisado por la firma y sin retribución monetaria. Esto está produciendo una suerte de autoexplotación voluntaria, libre y hasta inconsciente porque se invisibiliza la naturaleza laboral y productiva del trabajo no remunerado. Al grupo de trabajo libre, Arvidsson y Colleoni (2012) le suman todos aquellos seguidores que participan en el like-econom $y^{20}$ de las redes sociales. Estas "multitudes" abonan a la conformación de la sociedad del conocimiento que se nutre de lo que Moulier-Boutang (2011) denomina "conocimiento vivencial".

Las ideas generadas por las multitudes comienzan a fluir por el espacio virtual en franca desregulación. Cuando estas ideas están en una fase de mayor madurez, respaldan su autoría en esquemas regulatorios insertos en el movimiento copyleft, ${ }^{21}$ que ofrece una regulación más flexible que el copyright o propiedad intelectual. Este movimiento ha diseñado una plataforma virtual llamada Creative Commons, en la que los autores o propietarios de las innovaciones pueden optar por varios tipos de licencias: las que no permiten su uso comercial, las que autorizan su publicación y consulta, y las que permiten que se utilicen de manera comercial como base para hacer mejoras incrementales. Creative Commons hace alarde de ofrecer "contratos de licenciamiento", es decir, instrumentos de respaldo legal, accesibles y fáciles de operar para quienes producen de manera independiente ideas creativas que se colocan en la web con la finalidad de ser compartidas por las muchedumbres. $^{22}$

En el siguiente apartado se presenta la reconstrucción de los lanzamientos de las dos convocatorias masivas seleccionadas, que persiguen fines distintos pero ilustran la dinámica de producción y apropiación de valor al incorporar la participación activa del prosumidor en los procesos de innovación abierta. Estas formas inéditas de externalizar la producción de conocimiento han sido estrategias que han surgido en los dos capitalismos adjetivados que hemos expuesto: el netárquico y el cognitivo.

\section{Casos de crowdsourcing en los que "la creatividad compartida no tiene límite"23}

Para estudiar los dos casos de crowdsourcing que nos interesan, se retoma la propuesta de Mulgan (2006) de analizar la innovación como un proceso que integra tres fases: concepción, exploración y explotación, las cuales, en el modelo de innovación abierta, operan en espacios discontinuos. En la primera fase se conciben las ideas; se considera que ésta es la etapa más creativa y su respuesta es masiva. En la siguiente fase tiene lugar el proceso de exploración y acompañamiento de la evolución de la idea, se diseñan prototipos, se prueban las ideas y se formula el plan de negocios. En el último tramo de esta fase se desarrollan los procesos productivos, se analizan los cambios en materiales y en tecnología que una innovación conlleva para producirse de manera masiva. Es un momento en el que tiene que demostrarse la ventaja competitiva que la idea aporta a los planes de la firma (Atkins et al., 2000). La última fase es la de explotación. Aquí se pondera la viabilidad de los proyectos en términos de riesgo financiero, posicionamiento en el mercado y complejidad tecnológica. Esta fase da el "banderazo de salida” para que una innovación se concrete y esté disponible en el mercado.

20

Like es la expresión que cuantifica las preferencias de los consumidores. La traducción al español del concepto sería "economía del me gusta".

21 Este movimiento comenzó con producciones artísticas, como música y videos, que fluían con libertad en la web. Después abrió el respaldo legal a cualquier idea creativa.

22 Véase "State of the Commons 2016" (Creative Commons, 2016).

23 Tomado del título del artículo de Emprendices (2014). 

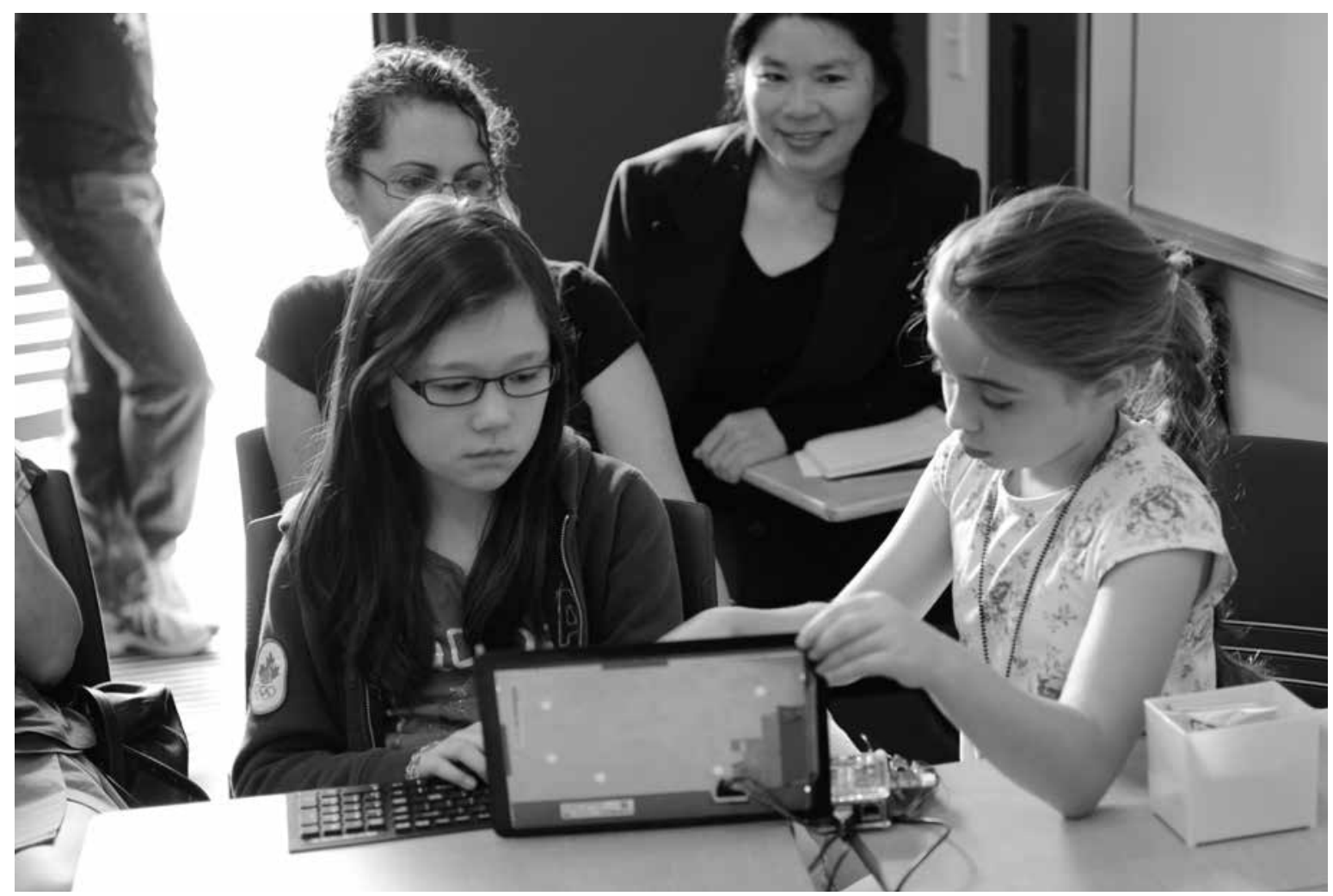

StePhen ChIn • Silicon Valley Code Camp, Raspberry Pi Gaming 4 Kids, octubre de 2013.

\section{Outsourcing en el sector automotriz}

A continuación, se presenta el lanzamiento de un crowdsourcing pionero por parte de una firma automotriz italiana. ${ }^{24}$ La planta de Fiat en Brasil lanzó en 2009 una convocatoria de crowdsourcing en Facebook y Twitter. ${ }^{25}$ La firma planeó durante tres años esta propuesta. Su propósito era "superar barreras, invitando a la gente a colaborar para crear un carro para el futuro". ${ }^{26}$ En un briefing, ${ }^{27}$ la convocatoria proponía lo siguiente:

Un automóvil compacto y ágil, confortable y seguro con soluciones innovadoras para el tráfico de las grandes ciudades, un motor libre de contaminantes y la capacidad de recibir actualizaciones
24 Las marcas que forman Fiat Group Automobiles son Fiat, Abarth, Alfa Romeo, Lancia y Fiat Professional. En fechas recientes, se asoció la firma estadounidense Chrysler.

25 Se escogió la marca Fiat porque implementó un cambio radical en una rama industrial que, en materia de innovación, ha sido muy cuidadosa y ha mantenido en total secrecía sus actividades relacionadas con las propuestas nuevas. Hay muchos otros proyectos de crowdsourcing en la industria automotriz, cabe mencionar la convocatoria de la camioneta todo terreno Tita PRO-4X, de Nissan, que registró todo el proceso en videos disponibles en YouTube, así como las convocatorias que lanza BMW, que se combinan con otra modalidad de innovación abierta, los fablabs, en los que se desarrollan prototipos de las ideas seleccionadas en el crowdsourcing. Este fablab en particular se llama BMw Guggenheim Lab. Para una definición más precisa de fablab, véase la nota 34.

26 Véase <http:www.ideaconnection.com/open-innovationsuccess/Fiat-Mio-the-World's-FirstCrowdsourced-Car-00 273.html>.

27 En un briefing se difunden las instrucciones y condiciones de participación. 
personalizadas, cambios de configuración y aportar interfaces entre el coche y el usuario. ${ }^{28}$

Lo que se puede observar en esta convocatoria es que propone un proyecto muy ambicioso y a la vez abierto a un espectro amplio de propuestas. En la página web en la que se registra el seguimiento de la convocatoria se hace hincapié en que se trata del primer automóvil diseñado con base en las ideas y necesidades de los usuarios, a partir de una invitación abierta. El proceso se documentó en el blog "Making Of Fiat Mio Blog", ${ }^{29}$ que además permitía la participación de seguidores. Este crowdsourcing tuvo una respuesta de 17000 participantes integrados en equipos, que ofrecieron 11000 propuestas, de las cuales sólo seis - $0.0005 \%$ - pasaron a la fase exploratoria del proceso innovador. Las propuestas pueden considerarse innovaciones incrementales que no alteran de manera significativa el modelo automotriz: llantas con una rotación de $90^{\circ}$ para estacionar el vehículo con facilidad, cámaras que sustituyen los espejos laterales y comunicación entre vehículos para evitar choques. También se tomaron en cuenta otras ideas, como el diseño de la carrocería, el logo y los colores exteriores. A los autores del número insignificante de propuestas que pasó a la fase exploratoria del proceso innovador del proyecto Fiat Mio, susceptibles de ser introducidas al producto que se lance al mercado, se les pidió que registraran la propiedad de su solución en Creative Commons bajo las condiciones de la firma. ${ }^{30} \mathrm{El}$ resto del diseño del automóvil fue ejecutado en coordinación con los proveedores que integran la cadena de valor de Fiat.

Todo el proceso tuvo un gran acompañamiento mediático y se resaltó la respuesta masiva del público. En el blog se introdujeron videos del proceso de fabricación de la propuesta final en la planta de Fiat en Minas Gerais. Los comentarios y likes ${ }^{31}$ para las ideas seleccionadas fueron muy apreciados por la firma. El resultado visible de este crowdsourcing fue un prototipo preliminar que se presentó al público en 2010, en la feria automotriz de São Paulo, un año después de que se lanzara la convocatoria. Se espera que el auto esté en el mercado en 2018. El proceso puede seguirse en tiempo real en los canales virtuales. ${ }^{32}$ Sin embargo, hay un proceso de selección de la información que se hace pública.

Los procesos estratégicos de la fase exploratoria permanecen invisibles y se procesan en el interior de la cadena de valor, como, por ejemplo, la sincronización de todas las partes ingenieriles y de negocios; la integración de los módulos, sus partes y componentes; el diseño de los procesos, el desarrollo de capacidades para su fabricación, la colaboración con proveedores, la factibilidad en el mercado, etc. Se trata de un proceso largo en el que es muy probable que una idea seleccionada se transforme y se convierta en una innovación serial que se diluye en el proceso, lo que deja en una posición marginal a los participantes del crowdsourcing.

A pesar de que las ideas captadas en el crowdsourcing tienen un impacto ínfimo sobre el producto final, en este caso se constata que su éxito radica en la táctica comunicativa con consumidores por medio

Véase "Fiat Mio, the World's First Crowdsourced Car" (IdeaConnection, 2011). De este sitio también se extrajo información del proceso. Véase <https://www.ideaconnection.com/blog/?s=Fiat+Mio>. La firma pidió la licencia que permite que las innovaciones tengan uso comercial como base para incorporar mejoras posteriores (Creative Commons, 2016).

31 Icono que representa la aprobación de lo que se muestra en alguna imagen o texto.

32 Para tener una idea de la visualización del proceso de participación de los prosumidores, se recomienda "Making Of Projeto Fiat Mio, Realidade Virtual”, <https://www.youtube. com/watch?v=Aot1vpD9Ou4>; "Fiat, Making Of Fiat Mio, Um carro colaborativo para o futuro", <https://www.youtube.com/ watch?v=3afCTtpQDD4>; Fiat, Making Of Projeto Fiat Mio, Episódio 12, Montagem do Modelo", <https://www.youtube. com/watch?v=FWTOvPa7COwFiat>; "Fiat, Making Of Proyecto Fiat Mio, Episodio 16, El Futuro de Fiat Mio, <https://www. youtube.com/watch?v=v1rkxRDpNXo>. 
de las redes sociales, lo que demuestra que se trata de una potente estrategia de mercado, ejemplo de lo que Lins Ribeiro llama "economía de la carnada" (en este volumen). Por tal motivo, toda la información disponible en la web sólo presenta la parte más entusiasta y motivacional del crowdsourcing, y en términos etnográficos, así se tiene que valorar. Algunos ejemplos de las declaraciones de los participantes son:

Recibí un tweet, ${ }^{33}$ entré al blog, ofrecí una propuesta sobre un material amigable con el medio ambiente.

Me gustó participar, aunque no aporté ninguna idea, vine a la Feria porque no es lo mismo verlo sólo en la pantalla de la computadora.

Yo ofrecí una idea, Mio es mío porque yo contribuí.

No se parece a nada más, es diferente porque el público entró en contacto con los profesionistas.

He seguido toda la aventura, ofrecí una idea y parece como si hubiese estado en la planta trabajando.

La firma reconoce públicamente, en la página web del crowdsourcing, el legado de datos aportado por las "multitudes". Afirma que la integración de comunidades creativas permite sortear las limitaciones de recursos y conocimiento, "literalmente pensar fuera de la caja". ${ }^{34}$ Se menciona que el producto final fue un esfuerzo compartido de capacidades internas y vinculación con proveedores para los módulos de mayor complejidad tecnológica. A esto se sumó la participación de espacios de cocreación, como incubadoras - muchas veces ancladas en las universidades-, startups, hubs y fablabs, ${ }^{35}$ en los que se reúnen jóvenes para integrar equipos multidisciplinarios. Se expresa también una serie de condiciones para aprovechar las ideas que vienen de los consumidores: la disponibilidad del personal de investigación y desarrollo de la firma para escuchar y colaborar con los consumidores, la capacidad administrativa de la firma para filtrar las propuestas factibles, sobre todo cuando se presentan miles de soluciones atractivas que en la práctica pueden ser contradictorias. ${ }^{36}$

Este caso de crowdsourcing automotriz expone que la innovación abierta puede ser una estrategia mercadológica, que permite sondear la aceptación de la marca y las preferencias del consumidor al echar a andar estrategias visuales del tipo reality show, ${ }^{37}$ en el que los consumidores proponen ideas que son absorbidas por la estructura de la firma en las fases de exploración y explotación, en las que el consumidor acepta con gusto convertirse en un simple seguidor del proceso.

33 Los tweets, o tuits, son ideas, noticias, comentarios cortos que se suben a una plataforma de microblogging y se difunden de manera masiva en tiempo real.

34 Véase <https://www.ennomotive.com/making-the-firstcrowdsourced-car-fiats-journey/>.

35 Los hubs, startups y fablabs son lugares cuya misión es generar ambientes para impulsar el talento y propiciar encuentros y colaboraciones entre emprendedores y otros actores sociales que contribuyen a la maduración de ideas. Las instalaciones están diseñadas bajo el paradigma de Google, esto significa que son espacios relajados que procuran un ambiente lúdico, de socialización y comunicación abierta e informal. Además, ofrecen a los emprendedores los servicios necesarios para su operación, como asistencia contable o legal, diseño de imagen, etc. Otra actividad de suma importancia es su función como centros de capacitación en los que se imparten de manera permanente cursos o pláticas para el desarrollo de habilidades empresariales. Estos centros se distinguen por ofrecer plataformas de acceso a redes de contacto en el plano global, para generar así una especie de club global que les da un sentido de pertenencia. Los fablabs cuentan con tecnología para desarrollar prototipos.

36 Véase "Making the First Crowdsourced Car: Fiat's Journey", $<$ https://www.ennomotive.com/making-the-first-crowdsour ced-car-fiats-journey/>.

37 El reality show, también llamado "espectáculo de la realidad", es un género de programa de televisión que difunde sucesos reales de personas comunes o celebridades sin guión preestablecido. Véase <https://definicion.de/reality-show/>. 


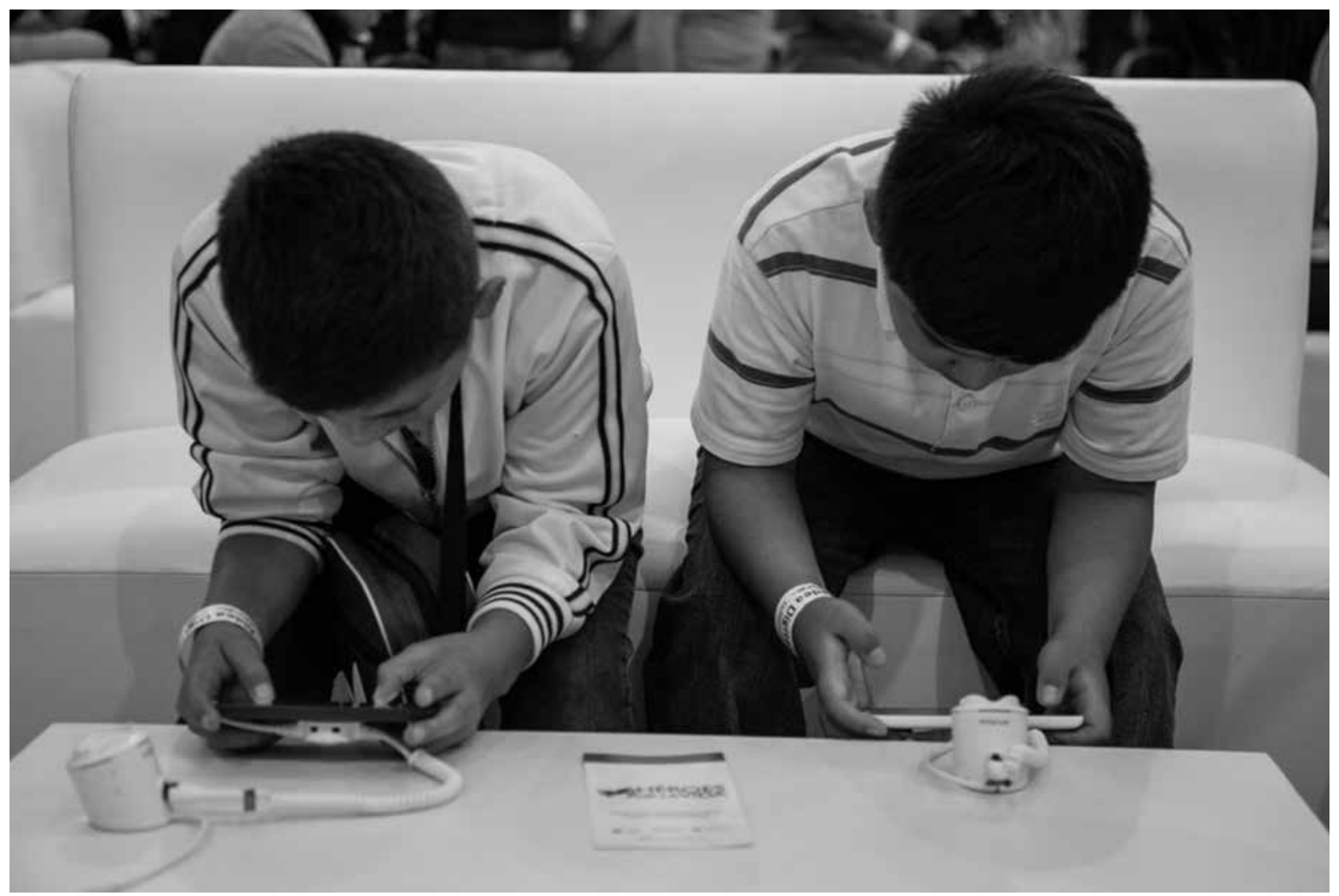

LABORATORIo PARa la CIUdAd • Espacio de videojuegos en la Aldea Digital Telmex 2015, Ciudad de México.

\section{Crowdsourcing en productos de limpieza $y$ aseo personal}

El segundo caso es el de Procter and Gamble (P\&G), en específico, el modelo de crowdsourcing llamado Connect \& Develop que opera desde 2001. La invitación que se difunde en la red es: "si tú tienes una innovación que puede mejorar las vidas de nuestros clientes, tú puedes ser nuestro próximo socio". ${ }^{38}$ Este crowdsourcing es un proyecto que aporta a las fortalezas de la firma: desarrollar diversos instrumentos para entender al consumidor y mantener importantes inversiones en innovación. ${ }^{39}$ En este rubro, $P \& G$ es pionera en promover la participación de externalidades en este proceso. En 2008, 50\% de las innovaciones se habían originado fuera de la empresa (Panduwawala et al., 2009). Otra de sus fortalezas es el incremento de su escala mediante la absoción de más marcas que se consumen en todo el mundo y perfeccionar sus líneas de distribución y logística. ${ }^{40}$

Connect \& Develop es una plataforma de innovación que se mantiene abierta al público. Uno de los propósitos de poner en práctica la innovación

Véase <http://www.pgconnectdevelop.com/>.

39 Panduwawala et al. (2009) mencionan que P\&G invirtió dos mil millones de dólares en innovaciones, el doble de lo que invirtió Unilever, su competidor más importante.

40 P\&G es una firma estadounidense que vende sus productos en 180 países y cuenta con 380 marcas, entre las más prestigiadas están Pampers, Pepto-Bismol, Crest, Vicks, Always, Oral B, Olay, Old Spice, Raun, Gillette, Pantene, Secret, Febreze, Dawn, Tide, Ariel, Charmin, Bounty y Pringles. Véase <http://www.pg.com/es_LATAM/MX/marcas-productos-p-and-g.shtml> 
abierta es "construir la confianza y la lealtad del consumidor a largo plazo" (Panduwawala et al., 2009). La convocatoria hace explícito el interés de la firma en "asociarse con las mentes más innovadoras a nivel mundial" (Panduwawala et al., 2009). La invitación incentiva el ofrecimiento de propuestas para el desarrollo de productos, procesos, tecnología, ventas virtuales y canales de distribución, en todos los productos que ofrece: cuidado personal, productos de belleza, productos para la limpieza del hogar, empaquetado. Lo que deja ver la plataforma es que P\&G ha optado por transformar su estructura organizativa y gobernanza para dar cabida a las ideas creativas que llegan por medio de la web. Este esquema de apertura para recibir propuestas de la muchedumbre pasa por un proceso de selección y sólo absorbe las que considera novedosas, en tanto que la firma se posiciona mejor en el mercado. ${ }^{41}$

Las propuestas son aceptadas por la plataforma Connect \& Develop después de que los emprendedores han registrado su idea en Creative Commons. ${ }^{42}$ Esto tiene varios propósitos: a la plataforma llegan propuestas más maduras, esto quiere decir que no se trata de lanzar ideas aún por desarrollar, como en el caso del crowdsourcing automotriz. De manera autogestionada, los emprendedores van concretando las propuestas, ya sea de manera autónoma, en espacios de cotrabajo o proyectos que desarrollaron en su formación profesional. La plataforma Creative Commons permite a los emprendedores respaldar el derecho de propiedad de la propuesta en caso de que sea atractiva para la firma. Al mismo tiempo, ésta se protege del riesgo de aceptar propuestas plagiadas o viceversa, de otros, incluso la competencia, que puedan piratear por no contar con licencias adecuadas de propiedad intelectual.

Connect \& Develop está incrustado en la estrategia corporativa para la innovación. Entre los programas que más se vinculan con la innovación abierta están los estudios que registran la experiencia del consumidor para detectar sus necesidades; un programa de venture capital — capital de riesgo- para apoyar la maduración de ideas originales que provienen de externalidades; una red interna, llamada Innovation net para promover sinergias entre lo que ellos llaman "empleados del conocimiento", que comparten información con quienes registran patentes y los que controlan el sistema de información de negocio. Esta red agiliza la comunicación interna y acota el ciclo de toma de decisiones para lanzar un nuevo producto (Panduwawala et al., 2009).

Además, con el propósito de incorporar de manera expedita propuestas creativas, se ha generado una estructura en red virtual con Innocentive, Yet2 y NineSigma. ${ }^{43}$ Esto permite multiplicar los canales de comunicación con los consumidores y echar mano de un equipo global cuya responsabilidad es "pescar talento", asesorar a los prospectos que son de interés para las marcas, presentar y negociar con las divisiones de la firma la pertinencia de una propuesta: "pastorear las innovaciones que mueven fronteras dentro de la compañía y dentro del mercado". ${ }^{44}$ Para esto, han surgido dos instancias críticas: los "busca talentos", que conectan la idea creativa con el cabildeo de la firma, y los "tutores", que operan en el back office ${ }^{45}$ de la idea creativa y contribuyen al desarrollo conceptual en la fase de exploración. Los autores citados afirman que se trata de un intrincado sistema que ha conformado un

41 Iniciativas que han surgido de innovadores mexicanos son Downy Libre Enjuague, Naturella, Pampers Básico y Vic Miel.

42 La plataforma virtual surge en 2001. En 2008 había 130 millones de ideas creativas registradas y su crecimiento es exponencial.

43 Plataformas vinculantes de innovación abierta que conectan firmas, emprendedores y fondeadoras.

44 Véase <http://www.pgconnectdevelop.com/what-is-con nect-develop>, página que publica las líneas de negocios abiertas para la cocreación en P\&G.

45 Tareas de respaldo de la gestión empresarial. Se traduce como "oficina de trastienda". 
“modelo espagueti de la innovación”. Algunos comentarios en blogs de participantes de crowdsourcings en estas plataformas, son: ${ }^{46}$

Es admirable que las marcas abran parte de sus procesos a la crowd.

Estas iniciativas son las que permiten entender que otro mundo es posible, y fomentando la interacción en los medios sociales podemos sentir que hemos aportado nuestro granito de arena.

Claro que sí, de eso se trata, de fomentar la participación de la gente para involucrarlos (de verdad) en los proyectos. Hay muchas personas con grandes ideas y por fin encuentran un modo de darles salida. ¡Gracias por tu comentario!

Me sigue impresionando la manera en que las grandes marcas aprovechan los Facebook para innovar y motivar a los consumidores a aportar sus ideas. Y me imagino que ésta es sólo una de las tantas estrategias novedosas que las empresas importantes utilizan para crear nuevos productos e ir siempre a la vanguardia.

Lo que se manifiesta en estos comentarios es la ausencia de intereses económicos de los participantes y más bien un sentimiento altruista y de inclusión.

Huston y Sakkab (2006) reconocen que la innovación abierta, para P\&G, es todavía un modelo inmaduro que está produciendo otras tensiones, como la necesidad de reasignar funciones a algunas áreas de la firma para la gestión masiva de propuestas o entrar en prácticas de cocreación con emprendedores. Estos autores calculan que de cada 100 ideas que provienen de externalidades, sólo una entra al mercado. Según Gassman, Sandmeier y Wecht (2006), 85\% del costo de la innovación se centra en la primera fase del proceso creativo, esto significa que la firma se libera de costos improductivos porque no absorbe ni invierte para generar un número significativo de ideas que no puede implementar. Las dos últimas fases del proceso innovador revisten una alta complejidad porque ahí subyace la sincronización de intereses y puntos de vista entre las divisiones de la firma. Huston y Sakkab (2006) denominan "la cultura de la ofensiva" al punto en el que la idea se incorpora al proceso interno de exploración. Ahí intervienen las metas y necesidades de las divisiones de investigación y desarrollo, manufactura, investigación de mercado, comercialización y finanzas. El crowdsourcing lanzado por P\&G mantiene un proceso de mayor secrecía en los tramos de exploración y explotación porque está más concentrado en "atrapar" ideas creativas que en sus proyectos publicitarios. Su consumo mundial es millonario. Hay que recordar que produce 380 marcas para una población consumidora de 5000 millones de personas en 160 países. $^{47}$

El crowdsourcing de P\&G tiene un propósito distinto al de Fiat; se trata de un espacio de cocreación en el que la firma obtiene ventajas directas en el proceso productivo, como son: acotar el ciclo de innovación, eliminar los costos por generación de conocimiento no aprovechado comercialmente y conocer las necesidades de los consumidores, además de contar con una cantidad exponencial de ideas de entre las cuales escoger. ¿Qué se puede aprender de las estrategias de crowdsourcing implementadas en el sector automotriz y en la producción de artículos de limpieza y aseo personal?

46 Se recomienda consultar los siguientes videos en YouTube: "Connect, and develop!", <https://www.youtube.com/watch?v =00g5W6oS4sM>; "Connect \& Develop Comp V1", <https: //www.youtube.com/watch?v=tBLpPbgEv4k>; "Connect + Develop MUM", <https://www.youtube.com/watch?v=NFNi 28spbik>; "Triad at Harvard - Connect \& Develop, o Novo P\&D”, <https://www.youtube.com/watch?v=JockuvJSVyQ>.

47 Véase <http://www.pg.com/es_LATAM/MX/marcas-p-and$\mathrm{g} /$ marcas-globales.shtml>. 


\section{A manera de conclusión: producción y expropiación de valor en la innovación abierta}

Las convocatorias de crowdsourcing se han convertido en espacios idóneos para la producción de valor. La identificación y fidelidad de los consumidores estimulan la participación de las multitudes y alimentan el prestigio y posicionamiento de la marca. Sin duda, esto contribuye a tejer puentes para atrapar las afinidades y fidelidades de los consumidores de la marca, lo que reproduce regímenes de valor "legibles", esto es, comprendidos y aceptados por emprendedores, consumidores, seguidores y empleados de las firmas. En este ensayo sobre los procesos de innovación abierta, se constata que la producción y expropiación de valor operan con estructuras y lógicas distintas en los dos espacios que se estudiaron. En ambos casos, se aprecia que las convocatorias anuales de Fiat Mio hacen aportaciones a la visión futura del transporte a partir de las preferencias de los consumidores. Connect \& Develop reconoce la importancia de capturar las demandas de un mercado segmentado, que refleja gustos diferenciados por género, edad y geografía.

La creación de valor tiene lugar en un espacio poroso, fluido, difuso, flexible y mediado por instrumentos tecnológicos, lo que da cabida a una estructura relacional horizontal, de total apertura, alimentada y reproducida por las comunidades de prosumidores y seguidores de las marcas. Por su parte, en el lanzamiento de las convocatorias, la firma exalta el valor de la creatividad, el talento de las muchedumbres creativas, la invitación abierta, el ambiente colaborativo que no impone condiciones a la participación masiva, sino al contrario, aporta un sentimiento de apertura de la cultura corporativa. Como afirma Benkler (2006), se ha generado un espacio incluyente, democrático y sin jerarquías, propicio para atraer la participación de jóvenes.

El lanzamiento de convocatorias no sería viable sin la mediación de las tecnologías de la información. Las redes sociales se han convertido en los conectores o mediadores que incentivan a los millennials a participar, ya no como espectadores, sino como sujetos involucrados el proceso. En el caso de Fiat Mio, se han aprovechado blogs y videos para comunicar de manera constante la evolución del proceso innovador y atrapar la atención de los seguidores de las propuestas. Además, los blogs son espacios propicios para la autorreproducción de procesos innovadores que fomentan la colaboración y el intercambio de ideas. Otra función importante de los blogs es su capacidad para almacenar el proceso secuencial de generación de conocimiento colectivo, lo que los convierte en una biblioteca virtual de libre acceso.

La minería de big data ${ }^{48}$ permite sacar provecho de la popularidad de las marcas. Las convocatorias de innovación abierta han propiciado la interacción entre la firma y sus seguidores por medio de likes y tweets, que se transforman en inversiones afectivas y se traducen en una referencia importante para calcular el valor financiero de la marca. Los crowdsourcings son una estrategia mediática muy potente para atrapar la participación masiva de la "clase creativa". Son mucho más efectivos que la simple publicidad, en la que no hay cabida para la interacción directa con la audiencia, pues al consumidor no sólo se le informa, sino que se le invita a intervenir de manera activa en el proceso.

En la serie de videos de Fiat Mio son evidentes los efectos seductores de las estrategias mercadológicas que se aprovechan de la pasión, la libertad, los valores sociales, la apertura, el altruismo, el placer y la creatividad. Los comentarios vertidos por los participantes en los blogs de este crowdsourcing reflejan que el interés de ganancia no es el objetivo

48 Es el análisis de grandes volúmenes de datos para descubrir patrones, en este caso, de preferencias de los consumidores. 


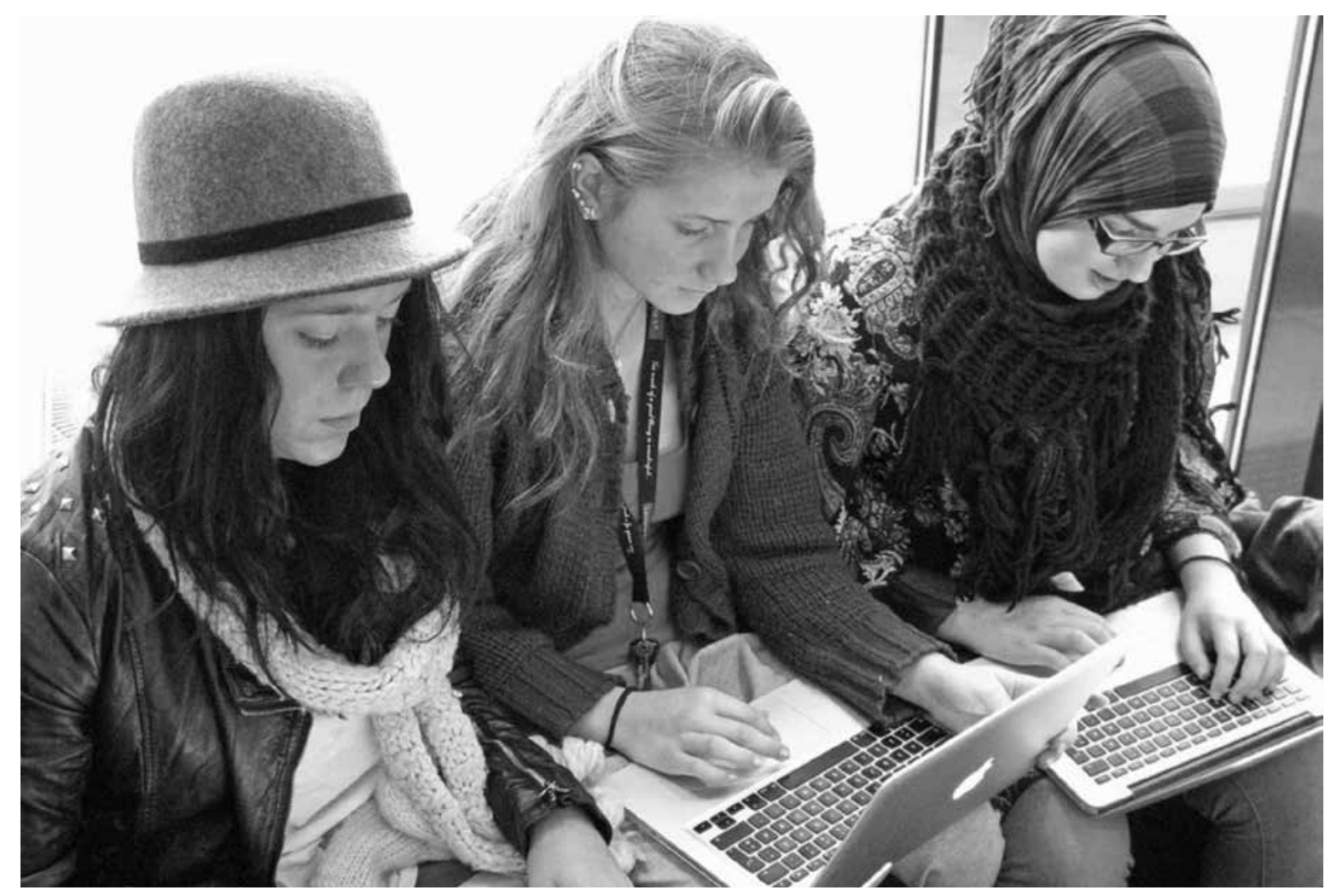

RAGNAM1211 - En la escuela Fyrtikkalleen, Noruega, todos los estudiantes de bachillerato usan su propia computadora portátil, septiembre de 2010.

de la participación de los prosumidores. Más bien, son respuestas espontáneas, voluntarias. Este estilo de producción de ideas creativas se percibe como una norma de vida, más que como una actividad productiva generadora de valor en el mercado. Los participantes voluntarios se respaldan en una filosofía del bien común, de la oportunidad de romper barreras institucionales para ofrecer a las grandes firmas ideas sobre sus gustos y preferencias. Esto reitera el papel central de la economía de los afectos en el capitalismo cognitivo. Los mensajes persuasivos y las expresiones de afiliación y lealtad generan un sentido de pertenencia que le otorga identidad incondicional a estos colectivos alrededor de la marca.

De esta manera, la firma construye un ambiente productivo en red, en el que el "trabajo" se autoorganiza y autorregula sin la intervención de relaciones o compromisos laborales. La economía del link ha permitido a las firmas apropiarse del valor producido de manera voluntaria por las multitudes creativas. Esto se combina con la llamada likeeconomy, reproducida por los seguidores que, con sólo pulsar un botón, envían información que se transforma en un activo para las firmas. Al tener lugar en espacios compartidos y colectivizados, esta like-economy produce un efecto de autopolinización como consecuencia de la intensidad de las interacciones en red (Moulier-Boutang, 2011).

El espacio abierto e incluyente que invita a la participación activa de los prosumidores sufre una metamorfosis para dar cabida a procesos de expropiación de propuestas creativas (Benkler, 2006: 108). Como se mencionó en la descripción de los casos, $P \& G$ es muy entusiasta en la manera en que 
Connect \& Develop se incrusta en la estructura organizativa de la firma; sin embargo, más allá del lanzamiento del crowdsourcing y los lineamientos claros de participación, el prosumidor no sabe qué sucede en el proceso de revisión y selección en el interior de la firma. De acuerdo con la información brindada por la firma, la abundancia de propuestas se somete a una suerte de embudo del que sólo 1\% pasa a una fase siguiente. No se sabe si el resto se tiró a la basura o se almacenó en el backlog de la empresa.

En el caso de Fiat Mio, el prosumidor o seguidor de la marca participa en la segunda fase del proceso innovador como espectador al seguir los videos de la incorporación de algunas ideas al diseño del automóvil que se difunden por YouTube. En la fase de exploración, sobre todo en la última fase denominada de explotación, predomina una estructura jerárquica que reduce la capacidad de agencia de los prosumidores a una posición marginal. En estas dos fases del proceso innovador, los prosumidores se introducen en un espacio incierto porque las firmas detentan el control de los datos que se vierten, así como la decisión de su uso y apropiación. Podría afirmarse que la innovación abierta vive un proceso de descontextualización en el plano virtual, que después se recontextualiza en el espacio de la firma para entrar en sintonía con los intereses y objetivos que la posicionan en el mercado.

Los procesos de innovación abierta arrancan con esquemas regulatorios indefinidos que vulneran el flujo de lo que Rifkin (2000) denomina "bienes comunes colaborativos". Cuando estas ideas maduran, los mismos prosumidores buscan el respaldo del movimiento de copyleft como una actividad autogestada para proteger la producción de ideas que aportan valor y fluyen con libertad en la web. En los dos casos de crowdsourcing analizados se aprecia que las firmas también sacan ventaja de estos instrumentos de protección de la creatividad, pues protegen sus propios intereses. Las ideas seleccionadas escrupulosamente bajo los criterios de la firma se convierten en ese momento en un bien que reporta valor y puede ser colocado en el mercado. Por ello la firma, al establecer como condición el registro de la propuesta en la plataforma virtual Creative Commons, se deslinda de cualquier responsabilidad de que las ideas aportadas por los prosumidores sean conocimiento pirateado o un remix ${ }^{49}$ de otra idea que prohíbe su utilización comercial.

Además de proteger los intereses de la firma, también se considera que es una manera de reducir tiempo y costos de transacción en procesos de negociación entre actores sociales tan disímbolos: por un lado, las grandes corporaciones con esquemas de gobernanza que responden a su liderazgo hegemónico, y por el otro, equipos de emprendedores cuyo único recurso negociador es aportar propuestas innovadoras que son atractivas para el posicionamiento de la marca. Al respecto, Huston y Sakkab (2006) argumentan que las innovaciones radicales son producto de innovaciones seriales. En una negociación sobre propiedad intelectual, las ideas lanzadas por los emprendedores se reducen a ser concebidas como un primer impulso que de manera obligada pasa por un proceso de ajuste o mutación como resultado de las tensiones y acomodamientos entre las divisiones de la firma. Éstas tienen que sincronizarse para ponderar los alcances y riesgos que implica lanzar un nuevo producto para consumo personal a un mercado mundial, lo cual exige instrumentos regulatorios más complejos y potentes que la protección de los contratos de licenciamiento de Creative Commons. ${ }^{50}$

Esta morfología dinámica y variable es la característica intrínseca de las redes de valor. Los dos casos expuestos constatan la vulnerabilidad de los creadores de ideas que se inspiran en estilos de vida diversos y

\footnotetext{
49 Remix es el resultado de la mezcla parcial de innovaciones. 50 La complejidad de este tema amerita un análisis jurídico relativo al derecho corporativo internacional.
} 
navegan en redes de valor sin una estructura organizativa que seleccione ex ante los recursos que se movilizarán, la programación de los procesos productivos, la asignación de obligaciones y compromisos a cumplir por todos los involucrados, sino que operan mediante un proceso continuo de ajuste experimental que se nutre de ideas que llegan por medio de una gran variedad de vías de contacto y cooperación con el consumidor. La estructura en red es aprovechada por las grandes firmas, que cuentan con los mecanismos para expropiar el valor del número infinito de propuestas creativas que circulan por el espacio de los flujos. D

\section{Bibliografía}

Arvidsson, Adam y Elanor Colleoni, 2012, "Value in Informational Capitalism and on the Internet", en The Information Society, vol. 28, núm. 3, pp. 135-150

Atkins, C. Richard, Paul Dykes, Janet Hagerty y Jeff Hoye, 2000, "How Customer Performance Partnerships Can Sharpen your Competitive Edge”, en Journal for Quality and Participation, vol. 23, núm. 3, pp. 35-38.

Benkler, Yochai, 2006, The Wealth of Networks, How Social Production Transforms Markets and Freedom, Yale University Press, New Haven. Brabham, Daren, 2010, "Moving The Crowd at Threadless. Motivations for Participation in a Crowdsourcing Application", en Information, Communication \& Society, vol. 13, núm. 8, pp. 1122-1145.

Callon, Michael, 1990, "Techno-Economic Networks and Irreversibility”, en The Sociological Review, vol. 38, supl. 1, pp. 132-161.

Castells, Manuel, 2006, La sociedad red: una visión global, Alianza, Madrid.

Chesbrough, Henry William, Wim Vanhaverbeke y Joel West (coords.), 2008, Open Innovation: Researching a New Paradigm, Oxford University Press, Oxford.

Christensen, Clayton M. y Michael Overdorf, 2000, “Meeting the Challenge of Disruptive Innovation”, en Harvard Business Review, marzoabril, pp. 66-76.

Desouza, Kevin, Yukika Awazu, Sanjeev Jha, Caroline Dombrowski, Sridhar Papagari, Peter Baloh y Jeffrey Y. Kim, 2008, "Costumer-Driven Innovation", en Research-Technology Management, vol. 51, núm. 3, pp. 35-44.

Florida, Richard, 2010, La clase creativa. La transformación de la cultura del trabajo y el ocio en el siglo xxı, Paidós, Barcelona.

Fumagalli, Andrea, 2010, Bioeconomía y capitalismo cognitivo. Hacia un nuevo paradigma de la acumulación, Traficantes de Sueños, Barcelona.

García Canclini, Néstor y Ernesto Piedras (coords.), 2013, Jóvenes creativos. Estrategias y redes culturales, Juan Pablos/Universidad Autónoma Metropolitana-Iztapalapa, México.

Gassmann, Oliver, Patricia Sandmeier y Christoph. H. Wecht, 2006, "Extreme Customer Innovation in the Front-End: Learning from a New Software Paradigm”, en International Journal of Technology Management, vol. 33, núm. 1, pp. 42-66.

Harvey, David, 2006, "La acumulación por desposesión”, en Carmen Bueno y Margarita Pérez Negrete (coords.), Espacios globales, Universidad Iberoamericana/Plaza y Valdés, México, pp. 21-53.

Hine, Christine, 2000, Etnografía virtual, Universitat Oberta de Catalunya, Barcelona.

Huston, Larry y Nabil Sakkab, 2006, “Connect and Develop: Inside Procter \& Gamble’s New Model for Innovation”, en Harvard Business Review, marzo.

Latour, Bruno, 2005, Reassembling the Social. An Introduction to Actor-Network Theory, Oxford University Press, Oxford.

Moulier-Boutang, Yann, 2011, Cognitive Capitalism, Polity Press, Cambridge.

Mulgan, Geoff, 2006, "The Process of Social Innovation”, en Innovations, MIT Press, primavera, pp. 145-162.

Ong, Aihwa y Stephen J. Collier, 2009, “Global Assemblages Anthropological Problems”, en Aihwa Ong y Stephen J. Collier (eds.), Global Assemblages Technology, Politics, and Ethics as Anthropological Problems, Blackwell, Massachusetts.

Panduwawala, Limali, Suvidha Venkatesh, Pedro Parraguez y Xiajing Zhang, 2009, “Connect and Develop. P\&G's Big Stake in Open Innovation", documento de trabajo, maestría en Innovation and Tecnology Management, 27 de noviembre, University of Bath, Bath. Disponible en línea: <http://www.openinnovate.co.uk/papers/PG_Open_Innovation.pdf>. 
Reichwald, Ralph, Sascha Seifert, David Walcher y Frank Piller, 2003, "Customers as Part of Value Webs: Towards a Framework for Webbed Customer Innovation Tools", documento de trabajo, Technische Universitaet Muenchen, Business School, Department of General and Industrial Management, Múnich.

Rifkin, Jeremy, 2000, La era del acceso. La revolución de la nueva economía, Paidós, México.

Ross, Andrew, 2001, “No Collar Labour, in America's 'New Economy”, en Socialist Register, núm. 37, pp. 77-87.

Toffler, Alvin, 1980, The Third Wave, Bantam Books, Nueva York.

Welker Marina, Damani Partridge y Rebecca Hardin, 2011, “Corporate Lives: New Perspectives on the Social Life of the Corporate Form”, en Current Anthropology, vol. 52, supl. 3, pp. 3-16.

Wittke, Volker y Heidemarie Hanekop (eds.), 2011, New Forms of Collaborative Innovation and Production on the Internet, Universitätsverlag Göttingen, Göttingen.

\section{Sitios web}

Connect + Develop, 2016. Disponible en línea: <http://www.pgconnectdevelop.com/>.

2016, "What is Connect + Develop?". Disponible en línea: <http://www.pgconnectdevelop.com/what-is-connect-develop>.

Creative Commons, 2016. Disponible en línea: <https://creativecommons.org/>.

Emprendices, 2014, "Crowdsourcing: donde la creatividad compartida no tiene límite", 8 de octubre. Disponible en línea: <https://www. emprendices.co/crowdsourcing-donde-la-creatividad-compartidano-tiene-limite/>.

Ennomotive, 2015, "Making the First Crowdsourced Car: Fiat's Journey”, 25 de enero. Disponible en línea: <https://www.ennomotive.com/ making-the-first-crowdsourced-car-fiats-journey/>.

IdeaConnection, 2011, "Fiat Mio, the World's First Crowdsourced Car", 28 de marzo. Disponible en línea: <https://www.ideaconnection.com/ open-innovation-success/Fiat-Mio-the-World\%27s-First-Crowdsourced-Car-00273.html>.

P\&G, 2017, "Nuestras marcas". Disponible en línea: <http://www.pg.com/es_LATAM/MX/marcas-p-and-g/marcas-globales.shtml>. 